\title{
Spinal Injections Versus Conservative Treatment for Spinal Pain: The Korean Spine Patient Outcomes Research Trial (K-SPORT) Observational Cohort
}

\author{
Minjeoung Kim², In Sun Park², Min A Lee ${ }^{3}$ Jinae Lee ${ }^{4}$, Jong Joo Lee ${ }^{5,6}$, Dong Ah Shin ${ }^{1,6 \bowtie}$ \\ ${ }^{1}$ Department of Neurosurgery, Yonsei University Severance Hospital, Seoul, Republic of Korea, \\ ${ }^{2}$ Yonsei Biomedical Research Institute, Yonsei University College of Medicine, Seoul, Republic of Korea, \\ ${ }^{3}$ Clinical Research Coordinator of Neurosurgery Department, Yonsei University Severance Hospital, Seoul, Republic of Korea, \\ ${ }^{4}$ Biometrics Division, DreamCIS, Ltd., Seoul, Republic of Korea, \\ ${ }^{5}$ Department of Neurosurgery, Bundan Jesaeng Hospital, Daejin Medical Center, Seongnam, Republic of Korea, \\ ${ }^{6}$ Department of Neurosurgery, Yonsei Spine Research Institute, Yonsei University College of Medicine, Seoul, Republic of Korea
}

\begin{abstract}
Objective: Spinal injections are increasingly used in the treatment of spinal pain, but its efficacy has been criticized. The purpose of this study was to compare the treatment effects of spinal injections and conservative care. Methods: This is a prospective observational cohort of nonsurgical candidates with spinal pain who were treated in one institute. Spinal injections included conventional injections, such as epidural steroid injection, selective nerve root block, medial branch block, interlaminar epidural block, caudal block, and sacroiliac joint block, and percutaneous epidural neuroplasty. Conservative treatment included medications, physical therapy, and exercise. Results: Primary outcome measures were changes from baseline in the visual analogue scale of lower back and leg pain (VAS_back and VAS_leg, respectively), 36-item Short Form Survey (SF-36), Euro Quality of Life-5 Dimensions (EQ-5D), and Oswestry Disability Index (ODI) at 1, 3, and 6 months. Secondary outcome that served as measure of clinical success was $50 \%$ (or 4-point) reduction from baseline VAS_back or VAS_leg and no increase from baseline ODI and SF36 or 30\% (or 2-point) reduction from baseline VAS_back or VAS_leg with 30\% (or 3-point) reduction in ODI from baseline or 30\% (or 20-point) increase from baseline SF36. Of the 142 patients followed up to 6 months, 47 patients underwent spinal injection and 95 patients underwent conservative treatment. The initial VA_leg, EQ-5D_mobility, EQ-5D_pain/discomfort, and SF36_bodily pain was higher in the injection group $(p=0.0309, p=0.0258, p=0.0042$, and $p=0.0474$ respectively). In both groups, spinal pain significantly decreased at 1 -month follow-up, and the effect was maintained for 6 months $(p<0.001)$. At all follow-up periods, there was no difference in the clinical success between the spinal injection and conservative treatment groups. Conclusion: The treatment effect was not different according to the diagnosis or disease severity. Both treatments were equally effective in carefully selected patients with spinal pain.
\end{abstract}

Key Words: K-SPORT; Spinal injection; Spinal pain

$\triangle$ Corresponding Author: Dong Ah Shin, Department of Neurosurgery, Yonsei Spine Research Institute, Yonsei University College of Medicine, 134 Shinchondong, Seodaemoon-Gu, Seoul 120-752, Republic of Korea Tel: +82-2-2228-2150, Fax: +82-02-393-9979, E-mail: cistern@yuhs.ac

\section{INTRODUCTION}

Low back pain with or without radiating pain is one of the common symptoms that most people experience at least once in a lifetime ${ }^{13)}$. According to the literature, the lifetime prevalence of low back pain is reported to be in the range of $51.0 \%$ to $84.0 \%{ }^{10,11,20)}$. In addition, the incidence of sciatica over 1 year is reported to be $9.3 \%^{6,17)}$. Particularly, after adjustment for methodological variation, the mean point prevalence of activity-limiting low back pain is $11.2 \pm 2.0 \%$ (standard de- viation), and the mean 1-month prevalence is estimated to be $23.2 \pm 2.9 \%{ }^{11)}$.

In most cases in which patients undergo appropriate treatment, low back pain decreases by $22 \%$ to $24 \%$ in 1 month and improves until 6 months ${ }^{24)}$. However, spinal pain makes it difficult for patients to return to their daily life and work, and the increase in the use of medical service causes an increase in economic cost.

Consequently, many types of intervention procedures are being performed to treat patients with spinal pain. In the United States, epidural injections increased by 99\% per 100,000 Medi- 
care beneficiaries, with an annual increase of 5\% from 2000 to $2014^{19)}$. Especially in Korea, various procedures are performed in addition to conventional spinal injection such as epidural steroid injection, selective nerve root block, medial branch block, and sacroiliac joint block. One of the various procedures performed in Korea is percutaneous epidural neu- roplasty, which is effective in single-level lumbar disc herniation ${ }^{12}$. However, the effectiveness of these spinal injections compared with that of conservative treatment remains controversial.

The aim of this study was to compare the treatment effects between spinal injections and conservative treatment for chronic spinal pain with symptom duration of a least 3 months prior to enrollment into the registry ${ }^{11)}$ using the 6-month follow-up result of the Korean Spine Patient Outcomes Research Trial (K-SPORT) based on the prospective observational cohorts. Spinal injection was superior to conservative treatment in the short term, but there was no significant difference in their treatment effects in the long term.

\section{MATERIALS AND METHODS}

\section{Study design and population}

Permission to conduct this study was granted by our Institutional Review Board (approval number: 4-2016-0462). This prospective observational cohort study was conducted from August 16, 2016, to October 30, 2018, as part of the K-SPORT by the National Research and Development Program of the Ministry of Health and Welfare of Korea. A total of 142 consecutive patients with visual analog scale (VAS) spinal pain score of $>5$ despite conservative treatments such as medications and physical therapies over 3 months at a single institution were included. For these patients, either spinal injection or conservative treatment was performed according to the patients' preference, and the clinical outcomes of both groups were investigated and analyzed at 1, 3, and 6 months. Diagnosis of lumbar spinal stenosis or herniated lumbar disc was made based on patients' symptoms, physical examination, and radiological findings on magnetic resonance imaging. Patients with neurological deficits and intractable pain at the time of diagnosis; those who were younger than 18 years; those who had previous lumbar spine surgery, vertebral fractures, spinal infections, tumors, inflammatory spinal diseases, pregnancy, or serious comorbidities at diagnosis; and those with gradually aggravating spinal pain or neurological symptoms that needed pain-alleviating procedure or surgery during the 3 months were excluded.

\section{Outcome assessment}

The baseline patients' characteristics including age, sex, weight, height, and comorbidities were prospectively collected and analyzed (Table 1). Spinal pain was assessed using VAS (scores range from 0 to 10 , with higher scores indicating more severe pain), and quality of life was assessed using the Euro Quality of Life-5 Dimensions (EQ-5D; scores range from 1 to 5 , with higher scores reflecting better quality of life) and the 36-item Short-Form Health Survey (SF-36, which address physical and mental subscales; scores range from 0 to 100 , with higher scores reflecting lower disability). The Oswestry Disability Index (ODI; scores range from 0 to 100, with higher scores reflecting more disability) was used to assess physical function. The primary outcomes were changes in VAS (back: VAS_back; leg: VAS_leg), SF-36, EQ-5D, and ODI scores from baseline to months 1, 3, and 6, as assessed after treatment of back and leg pain. The secondary outcome was the number of successful responders to treatment at each follow-up period. Successful response was defined depending on previous studies with some modifications ${ }^{5,7,16}$. Successful response was defined as $50 \%$ (or 4-point) reduction from baseline VAS_back or VAS leg and no increase from baseline SF-36 and ODI or 30\% (or 2-point) reduction from baseline VAS_back or VAS_leg with simultaneous $30 \%$ (or 3-point) reduction in ODI from baseline or $30 \%$ (or 20-point) increase in SF-36 from baseline.

\section{Conservative treatments and interventional procedures}

The conservative treatment used in this study included physical therapy, therapeutic exercise of the lower back, and medical treatment. Physical therapy included hot pack, ultrasound, and transcutaneous electrical nerve stimulation. Back exercise was systematic exercise that was conducted at an exercise clinic. Medical treatment was prescription of nonsteroidal anti-inflammatory drugs, anticonvulsants, antidepressants, muscle relaxants, and opioids, as necessary.

The spinal injections used in this study include transforaminal selective epidural steroid injections, medial branch block, caudal block, percutaneous epidural neuroplasty, balloon neuroplasty, and transforaminal epiduroscopic laser annuloplasty ${ }^{4,12,14,18,21,23)}$. The number of patients who underwent percutaneous epidural neuroplasty was the highest among the spinal injection group (1 month: 54, 70\%; 3 months: 46, 74\%; 6 months: 32, 68\%). Balloon neuroplasty, which induces epidural decompression and adhesiolysis, was performed using gentle side-to-side movement of the catheter with intermittent ballooning. Transforaminal epiduroscopic laser annuloplasty, a recent development, enables simultaneous decompression and thermal annuloplasty. Percutaneous epidural neuroplasty is a catheter procedure in which the catheter is placed directly into the lesion site compromising the nerve root. All conventional procedures were performed by one physician in compliance with the Spine Intervention Society protocol ${ }^{22}$. 


\section{Statistical analysis}

Statistical analyses were carried out using SAS version 9.4 (SAS Institute Inc., Cary, NC, USA). A $p$-value $<0.05$ was considered significant at two-tailed test. Continuous variables were presented as mean \pm standard deviation or median (Q1-Q3) depending on whether the normality assumption was met or not. If the normality assumption was not violated, then an independent two-sample $t$-test was adapted to compare the outcomes between the two groups. In case of violation of the normality assumption, the comparison was conducted using Mann-Whitney U-test. Categorical variables were compared using chi-squared test or Fisher's exact test, as appropriate, and the results were reported as frequency along with the percentage in parenthesis. Significant $p$-values at significance level

Table 1. Characteristics of the patients

\begin{tabular}{|c|c|c|c|}
\hline & Spinal injection $(n=47)$ & Conservative $(\mathrm{n}=95)$ & $p$-value ${ }^{a}$ \\
\hline Age & $58(44,64)$ & $56(41,64)$ & 0.5991 \\
\hline Weight & $64(55,76)$ & $60(53.95,71.5)$ & 0.1675 \\
\hline Height & $163.6(157,170)$ & $162(158,170.5)$ & 0.9716 \\
\hline Gender & & & 0.5941 \\
\hline Female & $24(51.06)$ & $44(46.32)$ & \\
\hline Male & $23(48.94)$ & $51(53.68)$ & \\
\hline \multicolumn{4}{|l|}{ Comorbidity } \\
\hline \multirow[t]{3}{*}{ Diabetes } & & & 0.8454 \\
\hline & $40(85.11)$ & $82(86.32)$ & \\
\hline & $7(14.89)$ & $13(13.68)$ & \\
\hline \multirow[t]{3}{*}{ Hypertension } & & & 0.6593 \\
\hline & $30(63.83)$ & $57(60)$ & \\
\hline & $17(36.17)$ & $38(40)$ & \\
\hline \multicolumn{4}{|l|}{ VAS } \\
\hline VAS Back & $6(4,8)$ & $5(3,7)$ & 0.0813 \\
\hline VAS Leg & $6(5,8)$ & $6(4,6)$ & 0.0309 \\
\hline \multicolumn{4}{|l|}{ EQ-5D } \\
\hline Mobility & $3(2,4)$ & $2(1,3)$ & 0.0258 \\
\hline Self care & $2(1,2)$ & $1(1,2)$ & 0.2638 \\
\hline Usual activity & $3(2,3)$ & $2(2,3)$ & 0.1697 \\
\hline Pain/Discomfort & $3(3,4)$ & $3(2,3)$ & 0.0042 \\
\hline Anxiety/Depression & $2(1,3)$ & $2(1,2)$ & 0.9294 \\
\hline VAS & $70(50,75)$ & $70(50,80)$ & 0.9669 \\
\hline \multicolumn{4}{|l|}{ SF-36 scores } \\
\hline PCS & $46.65 \pm 18.28$ & $50.15 \pm 19.22$ & 0.3014 \\
\hline General health & $53.62 \pm 17.71$ & $50.68 \pm 18.86$ & 0.3753 \\
\hline Physical function & $54.04 \pm 25.01$ & $57.53 \pm 22.91$ & 0.4097 \\
\hline Role-physical & $0(0,50)$ & $25(0,75)$ & 0.2038 \\
\hline Bodily pain & $22.5(22.5,57.5)$ & $45(22.5,67.5)$ & 0.0474 \\
\hline MCS & $53.81 \pm 16.53$ & $54.12 \pm 21$ & 0.9280 \\
\hline Vitality & $45(30,55)$ & $45(40,55)$ & 0.3557 \\
\hline Social functioning & $50(50,75)$ & $50(37.5,75)$ & 0.9877 \\
\hline Role-emotional & $66.67(0,100)$ & $66.67(0,100)$ & 0.8541 \\
\hline Mental health & $61.62 \pm 16.67$ & $61.01 \pm 18.6$ & 0.8503 \\
\hline ODI & $31.11(22.22,37.78)$ & $26.67(20,37.78)$ & 0.3091 \\
\hline
\end{tabular}

a: obtained from an independent two-sample $t$-test or Wilcoxon rank-sum test depending on satisfaction with normality assumption for continuous variables and a chi-square test or Fisher's exact test for categorical variables. 
of $\alpha=0.05$ are presented in boldface. For patients who finished up to 6 months of follow-up, we utilized a linear mixed model to investigate whether there are significant changes on the primary and secondary outcomes from baseline over time. Multiple comparisons were adjusted with Bonferroni method.

\section{RESULTS}

A total of 268 patients were enrolled in this study. Among them, 77 patients were excluded from the study due to crossover, that is, they switched treatment method from conservative treatment to spinal injection during the follow-up period. Consequently, 191 patients were included in this study, of whom 191 (spinal injection: 77; conservative: 114) had 1 month of follow-up, 166 (spinal injection: 62; conservative: 104) had 3 months of follow-up, and 142 (spinal injection: 47; conservative: 95) had 6 months of follow-up (Fig. 1).

There were no statistically significant differences in comorbid conditions, smoking history, physical examinations, symptoms, and severity of disease in patients who had 6 months of follow-up. In the baseline scores, the spinal injection group showed higher VAS_leg [6 (5-8) versus 6 (4-6); $p=0.0309]$, worse EQ-5D mobility, pain/discomfort [3 (2-4) versus $2(1-3) ; p=0.0258 ; 3$ (3-4) versus $3(2-3) ; p=0.0042]$, and worse SF-36 bodily pain [22.5 (22.5-57.5) versus 45.0 (22.5-67.5); $p=0.0474]$ (Table 1). This implies that patients who had worse pain were unintentionally included in the spinal injection group.

\section{Primary outcome}

There were statistically significant improvements after treatment in all categories of both groups at 6-month follow-up. VAS_leg was significantly more decreased in the spinal injection group than in the conservative treatment group at 1 month after the start of treatment (spinal injection group: $2.94 \pm 0.23$; conservative treatment group: $3.8 \pm 0.19$; $p=0.0096$ ). However, there was no statistical difference between the spinal injection and conservative treatment groups after 3 months. All EQ-5D scores gradually decreased until 3 months of follow-up and then increased again in the conservative treatment group. Similarly, the

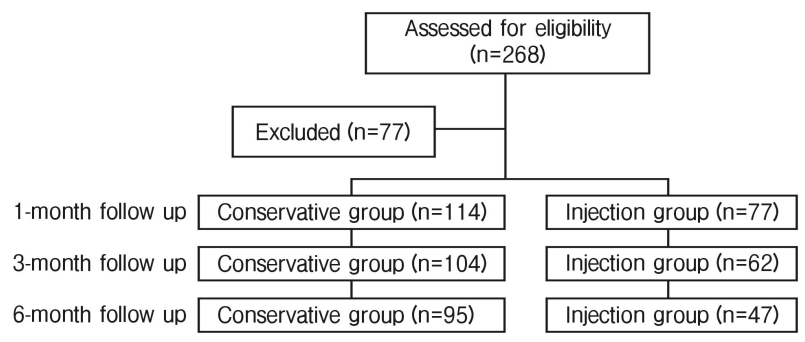

Fig. 1. Study flow diagram.
EQ-5D scores in the spinal injection group decreased until 1 month of follow-up and then increased again. The best therapeutic effect was obtained at 1 month in the spinal injection group and at 3 months in conservative treatment group. In both groups, the SF-36 and ODI scores decreased until 3 months of follow-up, showing improvement in clinical outcome and then increasing again (Fig. 2). In particular, the level of anxiety in the EQ-5D score was significantly lower in the spinal injection group at 1-month follow-up, but there was no significant difference between the two groups after 3-month follow-up.

\section{Secondary outcome}

The treatment results of the conservative and spinal injection groups were compared by two success criteria. According to criteria 1, $73(38 \%), 68(48 \%)$, and 57 patients (40\%) were successful responders at the 1-month, 3-month, and 6-month follow-up periods, respectively. According to criteria 2, 109 (57\%), $106(63 \%)$, and 85 patients (59\%) were successful responders at the 1-month, 3-month, and 6-month follow-up periods, respectively (Table 2). There was no statistically significant difference in the rate of successful responders between the spinal injection and conservative treatment groups for each follow-up period; however, the percentage of successful res-

Table 2. Rates of successful responders in spinal injection and conservative groups

\begin{tabular}{ccc}
\hline \hline $\begin{array}{c}\text { Follow-up } \\
\text { months }\end{array}$ & $\begin{array}{c}\text { Numbers of successful } \\
\text { responder criteria 1 }(\%)\end{array}$ & $\begin{array}{c}\text { Numbers of successful } \\
\text { responder criteria 2 }(\%)\end{array}$ \\
\hline $1(n=191)$ & $73(38)$ & $109(57)$ \\
$3(n=166)$ & $68(48)$ & $106(63)$ \\
$6(n=142)$ & $57(40)$ & $85(59)$ \\
\hline
\end{tabular}

criteria 1: 50\% (or_4-points) reduction from baseline VAS_back or VAS_leg and no increase from baseline SF-36 and ODI criteria 2: 30\% (or_2-points) reduction from baseline VAS_back or VAS_leg with any one of the following criteria: simultaneous_30\% (or_3-points) reduction in ODI from baseline, or _30\% (or_20-points) increase in SF-36 from baseline value

Table 3. Rates of successful responder among spinal injection and conservative group

Successful responders 1

Follow-up months Spinal injection (\%) Conservative (\%) p-value

\begin{tabular}{llll}
\hline 1 & $32(41.55)$ & $41(35.96)$ & 0.5296 \\
3 & $23(37.09)$ & $45(43.26)$ & 0.5358 \\
6 & $16(34.04)$ & $41(43.15)$ & 0.3893 \\
\hline
\end{tabular}

Successful responders 2

Follow-up months Spinal injection (\%) Conservative (\%) $p$-value

\begin{tabular}{llll}
\hline 1 & $49(63.63)$ & $60(52.63)$ & 0.1743 \\
3 & $42(67.74)$ & $64(61.53)$ & 0.5236 \\
6 & $25(53.19)$ & $60(63.15)$ & 0.3379 \\
\hline
\end{tabular}


ponders tended to be higher in the spinal injection group at 1 month (Table 3).

\section{DISCUSSION}

Previous studies have mainly focused on the comparison between surgical and nonsurgical treatments or among nonsurgical treatments of spinal pain ${ }^{4,14,30-32)}$. Thus, this prospective cohort study compared and analyzed the clinical outcomes between spinal injection and conservative treatment in patients with chronic spinal pain with a duration $>3$ months; results showed that spinal injection was superior to conservative treatment in the short term, but there was no significant difference their treatment effects in the long term.

There are some studies on the different types of spinal injection that are effective in the treatment of spinal pain. Carette et al. ${ }^{3)}$ reported that epidural steroid injection provides short-term (3-week) improvement of leg pain and sensory deficit in patients $(\mathrm{n}=158)$ with sciatica due to a herniated nucleus pulposus. Some studies reported that transforaminal epidural steroid injection and percutaneous epidural neuroplasty provided statistically significant improvement of lumbosacral radiculopathy ${ }^{25,30)}$.

Some studies investigated the effectiveness of conservative treatment of spinal pain and diversity of physical therapy methods. Shin et al. ${ }^{27)}$ reported that the clinical results of conservative treatment in patients with more than one-third spinal canal encroachment were satisfactory. Patients who underwent
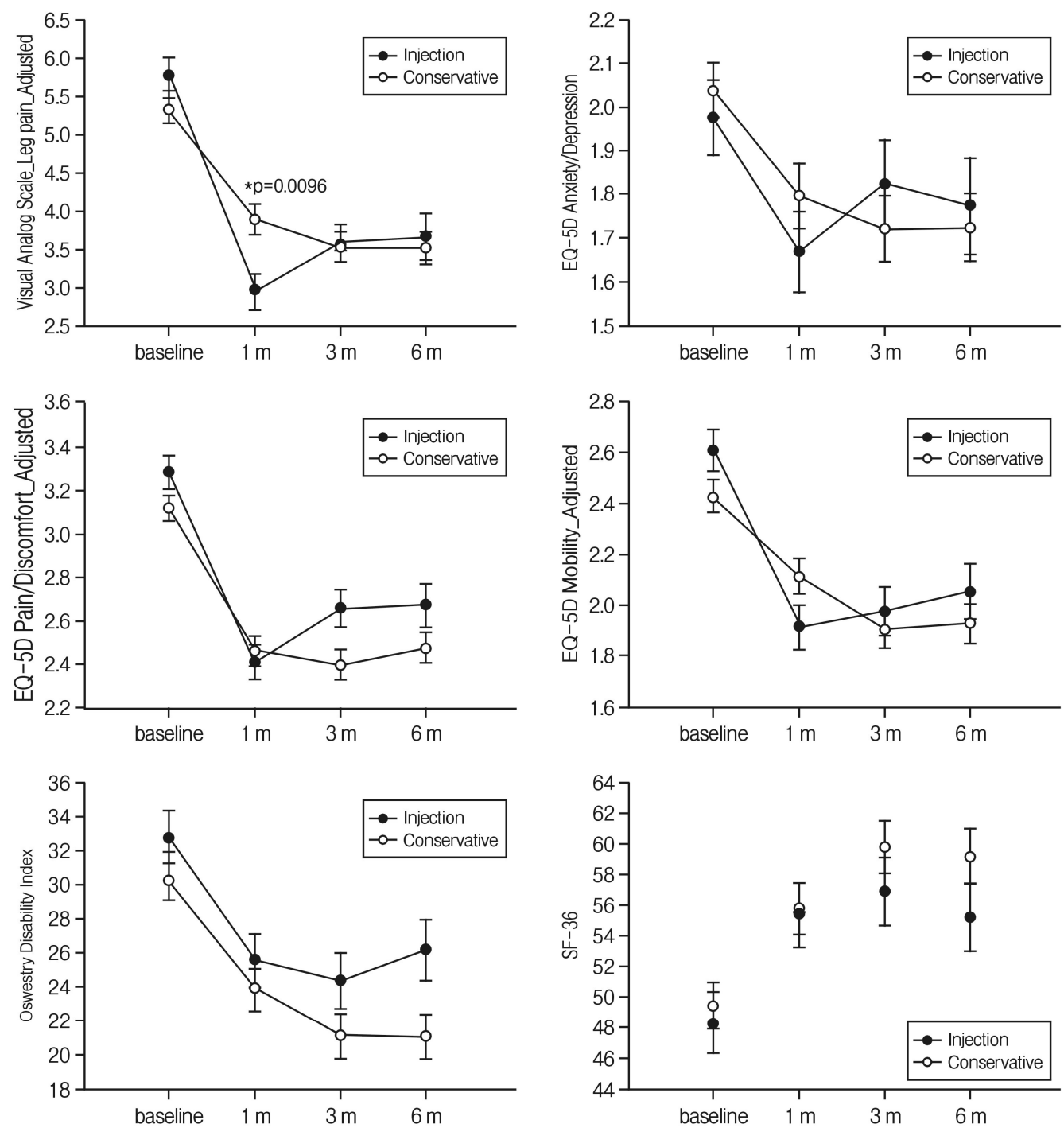

Fig. 2. Primary outcome (VAS-lower back pain, EQ-5D, SF-36, ODI) in the prospective observational cohort study during 1 month, 3 months and 6 months of follow-up. 
thermal mineral water therapy showed improvements in VAS for pain, ODI, EQ-5D, and SF-36 ${ }^{29}$. Some studies show that spinal manipulation, medication, massage, and exercise are effective for relieving chronic low back pain ${ }^{1,9,26}$. However, there was scant research on the superiority of conservative treatment to spinal injection.

Previous studies have reported that spinal injection and conservative treatment are both effective for spinal pain for up to 6 months, whereas the spinal injection provided better improvement in the short term ${ }^{2,3,15,25)}$. Similar to the findings of these studies, our results also showed that clinical outcome, including VAS, EQ-5D, and SF-36 scores, mostly improved in both groups until 6 months of follow-up. The spinal injection group showed a significant difference in VAS_leg at 1-month follow-up compared to the conservative treatment group. However, there was no statistical difference in VAS_leg between the two groups after 1 month. This result is similar to those of previous studies.

In many studies, spinal injection and conservative treatment can induce relief of low back and leg pain in the acute phase, whereas spinal injection provides better outcome in the short term $^{2,15)}$. Furthermore, the cost-effectiveness of spinal injection showed significantly good outcome in the acute phase only ${ }^{28)}$. However, the recent increase in the use of epidural steroid injection has led to an increase in medical expenses ${ }^{8}$.

\section{Study limitations}

The starting points of the clinical outcomes, especially VAS, differed. Patient who were in more pain and had worse functional performance preferred spinal injection. Therefore, the selection of treatment depends on patients' preference, and not on objective criteria. After enough provision of information of spinal injection and conservative treatment, including treatment costs, effectiveness and plan, the method of treatment was selected based on patients' preference.

The treatment method of both groups was heterogeneous. A large number of patients underwent percutaneous epidural neuroplasty. Furthermore, the medication prescribed for the conservative treatment group was not homogeneous, as the drugs were prescribed depending on the degree and pattern of patients' symptoms.

The primary and secondary outcomes of the spinal injection group were underestimated because crossover patients were excluded from this study. Crossover patients who switched their treatment method, from conservative treatment to spinal injection, imply failure of conservative treatment. In other words, the rate of successful conservative treatment may be overestimated.

\section{CONCLUSION}

Spinal injection was superior to conservative treatment in the short term. However, there was no significant difference in the treatment effect between the two groups in the long term. Therefore, it is appropriate to treat patients according to patients' preference of treatment methods.

\section{Funding}

This research was funded by the Korea Health Technology R\&D Project through the Korea Health Industry Development Institute (KHIDI), funded by the Ministry of Health \& Welfare, Republic of Korea, grant $\mathrm{HC} 15 \mathrm{C} 1320$.

\section{REFERENCES}

1. Andersson GB, Lucente T, Davis AM, Kappler RE, Lipton JA, Leurgans S: A comparison of osteopathic spinal manipulation with standard care for patients with low back pain. New England Journal of Medicine 341:1426-1431, 1999

2. Buchner M, Zeifang F, Brocai DR, Schiltenwolf M: Epidural corticosteroid injection in the conservative management of sciatica. Clinical Orthopaedics and Related Research 375:149-156, 2000

3. Carette S, Leclaire R, Marcoux S, Morin F, Blaise GA, St.-Pierre A, et al.: Epidural corticosteroid injections for sciatica due to herniated nucleus pulposus. New England Journal of Medicine 336:1634-1640, 1997

4. Center C, Relief P, Covington L, Parr AT: Caudal epidural injections in the management of chronic low back pain: A systematic appraisal of the literature. Pain Physician 15:E159-E198, 2012

5. Choi SS, Joo EY, Hwang BS, Lee JH, Lee G, Suh JH, et al.: A novel balloon-inflatable catheter for percutaneous epidural adhesiolysis and decompression. The Korean journal of pain 27: 178, 2014

6. Cook CE, Taylor J, Wright A, Milosavljevic S, Goode A, Whitford M: Risk factors for first time incidence sciatica: A systematic review. Physiotherapy Research International 19:65-78, 2014

7. Dworkin RH, Turk DC, Farrar JT, Haythornthwaite JA, Jensen MP, Katz NP, et al.: Core outcome measures for chronic pain clinical trials: Immpact recommendations. Pain 113:9-19, 2005

8. Friedly J, Chan L, Deyo R: Increases in lumbosacral injections in the medicare population: 1994 to 2001. Spine 32:1754-1760, 2007

9. Furlan AD, Brosseau L, Imamura M, Irvin E: Massage for low-back pain: A systematic review within the framework of the cochrane collaboration back review group. Spine 27:1896-1910, 2002

10. Henschke N, Kamper SJ, Maher CG: The epidemiology and economic consequences of pain: Mayo Clinic Proceedings: Elsevier, 2015, Vol 90, pp139-147

11. Hoy D, Bain C, Williams G, March L, Brooks P, Blyth F, et al.: A systematic review of the global prevalence of low back pain. Arthritis \& Rheumatism 64:2028-2037, 2012

12. Ji GY, Oh CH, Moon B, Choi SH, Shin DA, Yoon YS, et al.: Efficacy of percutaneous epidural neuroplasty does not correlate with dural sac cross-sectional area in single level disc disease. Yonsei Medical Journal 56:691-697, 2015

13. Kassebaum NJ, Arora M, Barber RM, Bhutta ZA, Brown J, Carter A, et al.: Global, regional, and national disability-adjusted lifeyears (dalys) for 315 diseases and injuries and healthy life expectancy (hale), 1990-2015: A systematic analysis for the global bur- 
den of disease study 2015. The Lancet 388:1603-1658, 2016

14. Kentucky L: Effectiveness of therapeutic lumbar transforaminal epidural steroid injections in managing lumbar spinal pain. Pain Physician 15:E199-E245, 2012

15. Koc Z, Ozcakir S, Sivrioglu K, Gurbet A, Kucukoglu S: Effectiveness of physical therapy and epidural steroid injections in lumbar spinal stenosis. Spine 34:985-989, 2009

16. Koh W, Choi S-S, Karm MH, Suh JH, Leem JG, Lee JD, et al.: Treatment of chronic lumbosacral radicular pain using adjuvant pulsed radiofrequency: A randomized controlled study. Pain Medicine 16:432-441, 2015

17. Konstantinou K, Dunn KM: Sciatica: Review of epidemiological studies and prevalence estimates. Spine 33:2464-2472, 2008

18. Manchikanti L, Singh V, Falco FJ, Cash KA, Pampati V: Effectiveness of thoracic medial branch blocks in managing chronic pain: A preliminary report of a randomized, double-blind controlled trial; clinical trial nct00355706. Pain Physician 11:491504, 2008

19. Manchikanti L, Pampati V, Hirsch JA: Retrospective cohort study of usage patterns of epidural injections for spinal pain in the us fee-for-service medicare population from 2000 to 2014. BMJ Open 6:e013042, 2016

20. Meucci RD, Fassa AG, Faria NMX: Prevalence of chronic low back pain: Systematic review. Revista de Saude Publica 49:73, 2015

21. Myong-Hwan Karm M, Choi S-S: Percutaneous epidural adhesiolysis using inflatable balloon catheter and balloon-less catheter in central lumbar spinal stenosis with neurogenic claudication: A randomized controlled trial. Pain Physician 21:593-605, 2018

22. Oh CH, Ji GY, Cho PG, Choi WS, Shin DA, Kim KN, et al.: The catheter tip position and effects of percutaneous epidural neuroplasty in patients with lumbar disc disease during 6-months of follow-up, 2014

23. Park CH, Lee SH: Endoscopic epidural laser decompression ver- sus transforaminal epiduroscopic laser annuloplasty for lumbar disc herniation: A prospective, randomized trial. Pain Physician 20:663-670, 2017

24. Pengel LH, Herbert RD, Maher CG, Refshauge KM: Acute low back pain: Systematic review of its prognosis. BMJ 327:323, 2003

25. Racz GB, Heavner JE, Raj PP: Percutaneous epidural neuroplasty: Prospective one-year follow-up. Pain Digest 9:97-102, 1999

26. Roelofs PD, Deyo RA, Koes BW, Scholten RJ, Van Tulder MW: Non-steroidal anti-inflammatory drugs for low back pain. Cochrane Database of Systematic Reviews, 2008

27. Shin B-J, Lee JC, Lee H-H, Jang H-D: Conservative treatment of lumbar disc herniation - A prospective study of disc herniation encroaching more than one-third of spinal canal. Journal of Korean Society of Spine Surgery 18:123-131, 2011

28. Spijker-Huiges A, Vermeulen K, Winters JC, van Wijhe M, van der Meer K: Costs and cost-effectiveness of epidural steroids for acute lumbosacral radicular syndrome in general practice: An economic evaluation alongside a pragmatic randomized control trial. Spine 39:2007-2012, 2014

29. Tefner IK, Nemeth A, Laszlofi A, Kis T, Gyetvai G, Bender T: The effect of spa therapy in chronic low back pain: A randomized controlled, single-blind, follow-up study. Rheumatol Int 32:31633169, 2012

30. Vad VB, Bhat AL, Lutz GE, Cammisa F: Transforaminal epidural steroid injections in lumbosacral radiculopathy: A prospective randomized study. Spine 27:11-15, 2002

31. Veihelmann A, Al Muderis M, Gollwitzer H: Percutaneous epidural lysis of adhesions in chronic lumbar radicular pain: A randomized, double-blind, placebo-controlled trial. Pain Physician 16:185-196, 2013

32. Zaina F, Tomkins-Lane C, Carragee E, Negrini S: Surgical versus non-surgical treatment for lumbar spinal stenosis. Cochrane Database of Systematic Reviews, 2016 